\title{
A parameterized complexity view on non-preemptively scheduling interval-constrained jobs: few machines, small looseness, and small slack
}

\author{
René van Bevern · Rolf Niedermeier · Ondřej Suchý
}

Submitted: August 7, 2015

Accepted: March 23, 2016

\begin{abstract}
We study the problem of non-preemptively scheduling $n$ jobs, each job $j$ with a release time $t_{j}$, a deadline $d_{j}$, and a processing time $p_{j}$, on $m$ parallel identical machines. Cieliebak et al (2004) considered the two constraints $\mid d_{j}-$ $\overline{t_{j} \mid \leq \lambda p_{j} \text { and } \mid} \overline{d_{j}-t_{j}} \mid \leq p_{j}+\sigma$ and showed the problem to be NP-hard for any $\lambda>1$ and for any $\sigma \geq 2$. We complement their results by parameterized complexity studies: we show that, for any $\lambda>1$, the problem remains weakly NPhard even for $m=2$ and strongly W[1]-hard parameterized by $m$. We present a pseudo-polynomial-time algorithm for constant $m$ and $\lambda$ and a fixed-parameter tractability result for the parameter $m$ combined with $\sigma$.
\end{abstract}

Keywords release times and deadlines - machine minimization $\cdot$ sequencing within intervals $\cdot$ shiftable intervals . fixed-parameter tractability $\cdot$ NP-hard problem

\section{Introduction}

Non-preemptively scheduling jobs with release times and deadlines on a minimum number of machines is a well-studied problem both in offline and online variants (Chen et al

René van Bevern is supported by grant 16-31-60007 mol_a_dk of the Russian Foundation for Basic Research (RFBR).

Ondřej Suchý is supported by grant 14-13017P of the Czech Science Foundation.

René van Bevern

Novosibirsk State University, Novosibirsk, Russian Federation, E-mail: rvb@nsu.ru

Rolf Niedermeier

Institut für Softwaretechnik und Theoretische Informatik, TU Berlin, Germany, E-mail: rolf.niedermeier@tu-berlin.de

Ondřej Suchý

Faculty of Information Technology, Czech Technical University in Prague, Prague, Czech Republic, E-mail: ondrej.suchy@ fit.cvut.cz
2016; Chuzhoy et al 2004; Cieliebak et al 2004; Malucelli and Nicoloso 2007; Saha 2013). In its decision version, the problem is formally defined as follows:

\section{INTERVAL-CONSTRAINED SCHEDULING}

Input: A set $J:=\{1, \ldots, n\}$ of jobs, a number $m \in \mathbb{N}$ of machines, each job $j$ with a release time $t_{j} \in \mathbb{N}$, a deadline $d_{j} \in \mathbb{N}$, and a processing time $p_{j} \in \mathbb{N}$.

Question: Is there a schedule that schedules all jobs onto $m$ parallel identical machines such that

1. each job $j$ is executed non-preemptively for $p_{j}$ time units,

2. each machine executes at most one job at a time, and

3. each job $j$ starts no earlier than $t_{j}$ and is finished by $d_{j}$.

For a job $j \in J$, we call the half-open interval $\left[t_{j}, d_{j}\right)$ its time window. A job may only be executed during its time window. The length of the time window is $d_{j}-t_{j}$.

We study INTERVAL-CONSTRAINED SCHEDULING with two additional constraints introduced by Cieliebak et al (2004). These constraints relate the time window lengths of jobs to their processing times:

Looseness If all jobs $j \in J$ satisfy $\left|d_{j}-t_{j}\right| \leq \lambda p_{j}$ for some number $\lambda \in \mathbb{R}$, then the instance has looseness $\lambda$. By $\lambda$ LOOSE INTERVAL-CONSTRAINED SCHEDULING we denote the problem restricted to instances of looseness $\lambda$.

Slack If all jobs $j \in J$ satisfy $\left|d_{j}-t_{j}\right| \leq p_{j}+\sigma$ for some number $\sigma \in \mathbb{R}$, then the instance has slack $\sigma$. By $\sigma$-SLACK INTERVAL-CONSTRAINED SCHEDULING we denote the problem restricted to instances of slack $\sigma$.

Both constraints on INTERVAL-CONSTRAINED SCHEDULING are very natural: clients may accept some small deviation of at most $\sigma$ from the desired start times of their jobs. Moreover, it is conceivable that clients allow for a larger deviation 
for jobs that take long to process anyway, leading to the case of bounded looseness $\lambda$.

Cieliebak et al (2004) showed that, even for constant $\lambda>$ 1 and constant $\sigma \geq 2$, the problems $\lambda$-LOOSE INTERVALCONSTRAINED SCHEDULING and $\sigma$-SLACK INTERVALCONSTRAINED SCHEDULING are strongly NP-hard.

Instead of giving up on finding optimal solutions and resorting to approximation algorithms (Chuzhoy et al 2004; Cieliebak et al 2004), we conduct a more fine-grained complexity analysis of these problems employing the framework of parameterized complexity theory (Cygan et al 2015; Downey and Fellows 2013; Flum and Grohe 2006; Niedermeier 2006), which so far received comparatively little attention in the field of scheduling with seemingly only a handful of publications (van Bevern et al 2015a,b; Bodlaender and Fellows 1995; Cieliebak et al 2004; Fellows and McCartin 2003; Halldórsson and Karlsson 2006; Hermelin et al 2015; Mnich and Wiese 2015). In particular, we investigate the effect of the parameter $m$ of available machines on the parameterized complexity of interval-constrained scheduling without preemption.

Related work INTERVAL-CONSTRAINED SCHEDULING is a classical scheduling problem and strongly NP-hard already on one machine (Garey and Johnson 1979, problem SS1). Besides the task of scheduling all jobs on a minimum number of machines, the literature contains a wide body of work concerning the maximization of the number of scheduled jobs on a bounded number of machines (Kolen et al 2007).

For the objective of minimizing the number of machines, Chuzhoy et al (2004) developed a factor- $O(\sqrt{\log n / \log \log n})$ approximation algorithm. Malucelli and Nicoloso (2007) formalized machine minimization and other objectives in terms of optimization problems in shiftable interval graphs. Online algorithms for minimizing the number of machines have been studied as well and we refer to recent work by Chen et al (2016) for an overview.

Our work refines the following results of Cieliebak et al (2004), who considered INTERVAL-CONSTR AINED SCHEDULING with bounds on the looseness and the slack. They showed that INTERVAL-CONSTRAINED SCHEDULING is strongly NP-hard for any looseness $\lambda>1$ and any slack $\sigma \geq$ 2 . Besides giving approximation algorithms for various special cases, they give a polynomial-time algorithm for $\sigma=1$ and a fixed-parameter tractability result for the combined parameter $\sigma$ and $h$, where $h$ is the maximum number of time windows overlapping in any point in time.

Our contributions We analyze the parameterized complexity of INTERVAL-CONSTRAINED SCHEDULING with respect to three parameters: the number $m$ of machines, the looseness $\lambda$, and the slack $\sigma$. More specifically, we refine known results of Cieliebak et al (2004) using tools of parameterized complexity analysis. An overview is given in Table 1.1.

In Section 3, we show that, for any $\lambda>1, \lambda$-LOOSE INTERVAL-CONSTRAINED SCHEDULING remains weakly NP-hard even on $m=2$ machines and that it is strongly W[1]hard when parameterized by the number $m$ of machines. In Section 4, we give a pseudo-polynomial-time algorithm for $\lambda$ LOOSE INTERVAL-CONSTRAINED SCHEDULING for each fixed $\lambda$ and $m$. Finally, in Section 5, we give a fixed-parameter algorithm for $\sigma$-SLACK INTERVAL-CONSTRAINED SCHEDULING when parameterized by $m$ and $\sigma$. This is in contrast to our result from Section 3 that the parameter combination $m$ and $\lambda$ presumably does not give fixed-parameter tractability results for $\lambda$-LOOSE INTERVAL-CONSTRAINED SCHEDULING.

\section{Preliminaries}

Basic notation We assume that $0 \in \mathbb{N}$. For two vectors $\mathbf{u}=$ $\left(u_{1}, \ldots, u_{k}\right)$ and $\mathbf{v}=\left(v_{1}, \ldots, v_{k}\right)$, we write $\mathbf{u} \leq \mathbf{v}$ if $u_{i} \leq v_{i}$ for all $i \in\{1, \ldots, k\}$. Moreover, we write $\mathbf{u} \supsetneqq \mathbf{v}$ if $\mathbf{u} \leq \mathbf{v}$ and $\mathbf{u} \neq \mathbf{v}$, that is, $\mathbf{u}$ and $\mathbf{v}$ differ in at least one component. Finally, $\mathbf{1}^{k}$ is the $k$-dimensional vector consisting of $k 1$-entries.

Computational complexity We assume familiarity with the basic concepts of NP-hardness and polynomial-time manyone reductions (Garey and Johnson 1979). We say that a problem is (strongly) $C$-hard for some complexity class $C$ if it is $C$-hard even if all integers in the input instance are bounded from above by a polynomial in the input size. Otherwise, we call it weakly C-hard.

In the following, we introduce the basic concepts of parameterized complexity theory, which are in more detail discussed in corresponding text books (Cygan et al 2015; Downey and Fellows 2013; Flum and Grohe 2006; Niedermeier 2006).

Fixed-parameter algorithms The idea in fixed-parameter algorithmics is to accept exponential running times, which are seemingly inevitable in solving NP-hard problems, but to restrict them to one aspect of the problem, the parameter.

Thus, formally, an instance of a parameterized problem $\Pi$ is a pair $(x, k)$ consisting of the input $x$ and the parameter $k$. A parameterized problem $\Pi$ is fixed-parameter tractable (FPT) with respect to a parameter $k$ if there is an algorithm solving any instance of $\Pi$ with size $n$ in $f(k) \cdot \operatorname{poly}(n)$ time for some computable function $f$. Such an algorithm is called a fixed-parameter algorithm. It is potentially efficient for small values of $k$, in contrast to an algorithm that is merely running in polynomial time for each fixed $k$ (thus allowing the degree of the polynomial to depend on $k$ ). FPT is the complexity class of fixed-parameter tractable parameterized problems. 
Table 1.1 Overview of results on INTERVAL-CONSTRAINED SCHEDULING for various parameter combinations. The parameterized complexity with respect to the combined parameter $\lambda+\sigma$ remains open.

\begin{tabular}{|c|c|c|c|}
\hline \multirow{2}{*}{$\begin{array}{r}\text { Combined } \\
\text { with }\end{array}$} & \multicolumn{3}{|r|}{ Parameter } \\
\hline & looseness $\lambda$ & slack $\sigma$ & number $m$ of machines \\
\hline$\lambda$ & $\begin{array}{l}\text { NP-hard for any } \lambda>1 \\
\text { (Cieliebak et al 2004) }\end{array}$ & $?$ & $\begin{array}{l}\text { W[1]-hard for parameter } m \text { for any } \lambda>1 \text { (Theorem 3.1), } \\
\text { weakly NP-hard for } m=2 \text { and any } \lambda>1 \text { (Theorem } 3.1 \text { ), } \\
\text { pseudo-polynomial time for fixed } m \text { and } \lambda \text { (Theorem 4.1) }\end{array}$ \\
\hline$\sigma$ & & $\begin{array}{l}\text { NP-hard for any } \sigma \geq 2 \\
\text { (Cieliebak et al 2004) }\end{array}$ & fixed-parameter tractable for parameter $\sigma+m$ (Theorem 5.1) \\
\hline$m$ & & & NP-hard for $m=1$ (Garey and Johnson 1979) \\
\hline
\end{tabular}

We refer to the sum of parameters $k_{1}+k_{2}$ as the combined parameter $k_{1}$ and $k_{2}$.

Parameterized intractability To show that a problem is presumably not fixed-parameter tractable, there is a parameterized analog of NP-hardness theory. The parameterized analog of NP is the complexity class W[1] $\supseteq$ FPT, where it is conjectured that FPT $\neq \mathrm{W}[1]$. A parameterized problem $\Pi$ with parameter $k$ is called W[1]-hard if $\Pi$ being fixed-parameter tractable implies W[1] = FPT. W[1]-hardness can be shown using a parameterized reduction from a known W[1]-hard problem: a parameterized reduction from a parameterized problem $\Pi_{1}$ to a parameterized problem $\Pi_{2}$ is an algorithm mapping an instance $I$ with parameter $k$ to an instance $I^{\prime}$ with parameter $k^{\prime}$ in time $f(k) \cdot \operatorname{poly}(|I|)$ such that $k^{\prime} \leq g(k)$ and $I^{\prime}$ is a yes-instance for $\Pi_{1}$ if and only if $I$ is a yes-instance for $\Pi_{2}$, where $f$ and $g$ are arbitrary computable functions.

\section{A strengthened hardness result}

In this section, we strengthen a hardness result of Cieliebak et al (2004), who showed that $\lambda$-LOOSE INTER VAL-CONSTRAINED SCHEDULING is NP-hard for any $\lambda>1$. This section proves the following theorem:

Theorem 3.1 Let $\lambda: \mathbb{N} \rightarrow \mathbb{R}$ be such that $\lambda(n) \geq 1+n^{-c}$ for some integer $c \geq 1$ and all $n \geq 2$.

Then $\lambda(n)$-LoOsE INTERVAL-CONSTRAINED SCHEDULING of $n$ jobs on m machines is

(i) weakly NP-hard for $m=2$, and

(ii) strongly W[1]-hard for parameter $m$.

Note that Theorem 3.1, in particular, holds for any constant function $\lambda(n)>1$.

We remark that Theorem 3.1 cannot be proved using the NP-hardness reduction given by Cieliebak et al (2004), which reduces 3 -SAT instances with $k$ clauses to INTERVAL-CONSTRAINED SCHEDULING instances with $m=3 k$ machines. Since 3-SAT is trivially fixed-parameter tractable for the parameter number $k$ of clauses, the reduction of Cieliebak

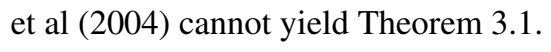

Instead, to prove Theorem 3.1 , we give a parameterized polynomial-time many-one reduction from BIN PACKING with $m$ bins and $n$ items to $\lambda(m n)$-LOOSE INTERVAL-CONSTRAINED SCHEDULING with $m$ machines and $m n$ jobs.

\section{BIN PACKING}

Input: A bin volume $V \in \mathbb{N}$, a list $a_{1}, \ldots, a_{n} \in \mathbb{N}$ of items, and a number $m \leq n$ of bins.

Question: Is there a partition $S_{1} \uplus \cdots \uplus S_{m}=\{1, \ldots, n\}$ such that $\sum_{i \in S_{k}} a_{i} \leq V$ for all $1 \leq k \leq m$ ?

Since BIN PACKING is weakly NP-hard for $m=2$ bins and $\mathrm{W}$ [1]-hard parameterized by $m$ even if all input numbers are polynomial in $n$ (Jansen et al 2013), Theorem 3.1 will follow.

Our reduction, intuitively, works as follows: for each of the $n$ items $a_{i}$ in a BIN PACKING instance with $m$ bins of volume $V$, we create a set $J_{i}:=\left\{j_{i}^{1}, \ldots, j_{i}^{m}\right\}$ of $m$ jobs that have to be scheduled on $m$ mutually distinct machines. Each machine represents one of the $m$ bins in the BIN PACKING instance. Scheduling job $j_{i}^{1}$ on a machine $k$ corresponds to putting item $a_{i}$ into bin $k$ and will take $B+a_{i}$ time of machine $k$, where $B$ is some large integer chosen by the reduction. If $j_{i}^{1}$ is not scheduled on machine $k$, then a job in $J_{i} \backslash\left\{j_{i}^{1}\right\}$ has to be scheduled on machine $k$, which will take only $B$ time of machine $k$. Finally, we choose the latest deadline of any job as $n B+V$. Thus, since all jobs have to be finished by time $n B+V$ and since there are $n$ items, for each machine $k$, the items $a_{i}$ for which $j_{i}^{1}$ is scheduled on machine $k$ must sum up to at most $V$ in a feasible schedule. This corresponds to satisfying the capacity constraint of $V$ of each bin.

Formally, the reduction works as follows and is illustrated in Figure 3.1.

Construction 3.2 Given a BIN PACKING instance $I$ with $n \geq 2$ items $a_{1}, \ldots, a_{n}$ and $m \leq n$ bins, and $\lambda: \mathbb{N} \rightarrow \mathbb{R}$ such that $\lambda(n) \geq 1+n^{-c}$ for some integer $c \geq 1$ and all $n \geq 2$, we construct an INTERVAL-CONSTRAINED SCHEDULING 


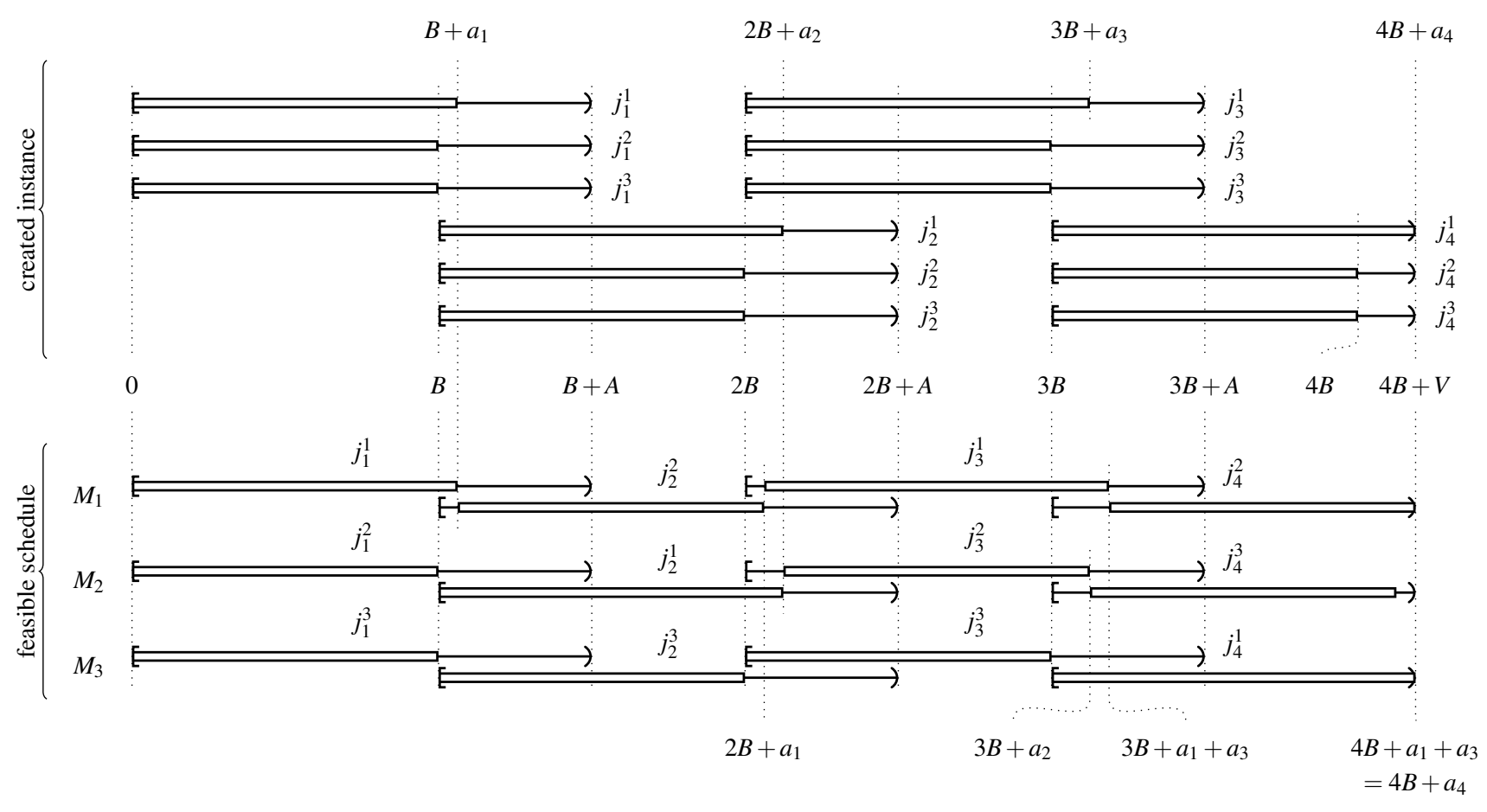

Fig. 3.1 Reduction from BIN PACKING with four items $a_{1}=1, a_{2}=a_{3}=2, a_{4}=3$, bin volume $V=3$, and $m=3$ bins to $3 / 2$-LOOSE INTERVALConstrained Scheduling. That is, Construction 3.2 applies with $c=1, A=8$, and $B=3 \cdot 4 \cdot 8=96$. The top diagram shows (not to scale) the jobs created by Construction 3.2. Herein, the processing time of each job is drawn as a rectangle of corresponding length in an interval being the job's time window. The bottom diagram shows a feasible schedule for three machines $M_{1}, M_{2}$, and $M_{3}$ that corresponds to putting items $a_{1}$ and $a_{3}$ into the first bin, item $a_{2}$ into the second bin, and $a_{4}$ into the third bin.

instance with $m$ machines and $m n$ jobs as follows. First, let

$A:=\sum_{i=1}^{n} a_{i} \quad$ and $\quad B:=(m n)^{c} \cdot A \geq 2 A$.

If $V>A$, then $I$ is a yes-instance of BIN PACKING and we return a trivial yes-instance of INTERVAL-CONSTRAINED SCHEDULING.

Otherwise, we have $V \leq A$ and construct an instance of INTERVAL-CONSTRAINED SCHEDULING as follows: for each $i \in\{1, \ldots, n\}$, we introduce a set $J_{i}:=\left\{j_{i}^{1}, \ldots, j_{i}^{m}\right\}$ of jobs. For each job $j \in J_{i}$, we choose the release time

$$
t_{j}:=(i-1) B
$$

the processing time

$p_{j}:= \begin{cases}B+a_{i} & \text { if } j=j_{i}^{1}, \\ B & \text { if } j \neq j_{i}^{1},\end{cases}$

and the deadline

$d_{j}:= \begin{cases}i B+A & \text { if } i<n, \\ i B+V & \text { if } i=n .\end{cases}$

This concludes the construction.
Remark 3.3 Construction 3.2 outputs an INTERVAL-CONSTRAINED SCHEDULING instance with agreeable deadlines, that is, the deadlines of the jobs have the same relative order as their release times. Thus, in the offline scenario, all hardness results of Theorem $\underline{3.1}$ will also hold for instances with agreeable deadlines.

In contrast, agreeable deadlines make the problem significantly easier in the online scenario: Chen et al (2016) showed an online-algorithm with constant competitive ratio for INTERVAL-CONSTRAINED SCHEDULING with agreeable deadlines, whereas there is a lower bound of $n$ on the competitive ratio for general instances (Saha 2013).

In the remainder of this section, we show that Construction 3.2 is correct and satisfies all structural properties that allow us to derive Theorem $\underline{3.1}$.

First, we show that Construction 3.2 indeed creates an INTERVAL-CONSTRAINED SCHEDULING instance with small looseness.

Lemma 3.4 Given a BIN PACKING instance with $n \geq 2$ items and $m$ bins, Construction 3.2 outputs an INTERVAL-CONSTRAINED SCHEDULING instance with

(i) at most m machines and mn jobs and

(ii) looseness $\lambda(m n)$.

Proof It is obvious that the output instance has at most $m n$ jobs and $m$ machines and, thus, (i) holds. 
Towards (ii), observe that $m n \geq n \geq 2$, and hence, for each $i \in\{1, \ldots, n\}$ and each job $j \in J_{i}, \underline{(3.1)}$ yields

$$
\begin{aligned}
\frac{\left|d_{j}-t_{j}\right|}{p_{j}} & \leq \frac{(i B+A)-(i-1) B}{B}=\frac{B+A}{B}=1+\frac{A}{B} \\
& =1+\frac{A}{(m n)^{c} \cdot A}=1+(m n)^{-c} \leq \lambda(m n) .
\end{aligned}
$$

We now show that Construction 3.2 runs in polynomial time and that, if the input BIN PACKING instance has polynomially bounded integers, then so has the output INTERVAL-CONSTRAINED SCHEDULING instance.

Lemma 3.5 Let I be a BIN PACKING instance with $n \geq$ 2 items $a_{1}, \ldots, a_{n}$ and let $a_{\max }:=\max _{1 \leq i \leq n} a_{i}$. Construction 3.2 applied to $I$

(i) runs in time polynomial in $|I|$ and

(ii) outputs an INTERVAL-CONSTRAINED SCHEDULING instance whose release times and deadlines are bounded by a polynomial in $n+a_{\max }$.

Proof We first show (ii), thereafter we show (i).

(ii) It is sufficient to show that the numbers $A$ and $B$ in Construction 3.2 are bounded polynomially in $n+a_{\max }$ since all release times and deadlines are computed as sums and products of three numbers not larger than $A, B$, or $n$. Clearly, $A=\sum_{i=1}^{n} a_{i} \leq n \cdot \max _{1 \leq i \leq n} a_{i}$, which is polynomially bounded in $n+a_{\max }$. Since $m n \leq n^{2}$, also $B=(m n)^{c} \cdot A$ is polynomially bounded in $n+a_{\max }$.

(i) The sum $A=\sum_{1=1}^{n} a_{i}$ is clearly computable in time polynomial in the input length. It follows that also $B=(m n)^{c}$. $A$ is computable in polynomial time.

It remains to prove that Construction 3.2 maps yes-instances of BIN PACKING to yes-instances of INTERVAL-CONSTRAINED SCHEDULING, and no-instances to no-instances.

Lemma 3.6 Given a BIN PACKING instance I with $m$ bins and the items $a_{1}, \ldots, a_{n}$, Construction 3.2 outputs an INTERVAL-CONSTRAINED SCHEDULING instance $I^{\prime}$ that is a yes-instance if and only if I is.

Proof $(\Rightarrow)$ Assume that $I$ is a yes-instance for BIN PACKING. Then, there is a partition $S_{1} \uplus \cdots \uplus S_{m}=\{1, \ldots, n\}$ such that $\sum_{i \in S_{k}} a_{i} \leq V$ for each $k \in\{1, \ldots, m\}$. We construct a feasible schedule for $I^{\prime}$ as follows. For each $i \in\{1, \ldots, n\}$ and $k$ such that $i \in S_{k}$, we schedule $j_{i}^{1}$ on machine $k$ in the interval

$$
\left[(i-1) B+\sum_{j \in S_{k}, j<i} a_{j} \quad, \quad i B+\sum_{j \in S_{k}, j<i} a_{j}+a_{i}\right)
$$

and each of the $m-1$ jobs $J_{i} \backslash\left\{j_{i}^{1}\right\}$ on a distinct machine $\ell \in$ $\{1, \ldots, m\} \backslash\{k\}$ in the interval

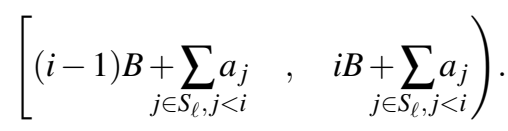

It is easy to verify that this is indeed a feasible schedule.

$(\Leftarrow)$ Assume that $I^{\prime}$ is a yes-instance for INTERVAL-CONSTRAINED SCHEDUling. Then, there is a feasible schedule for $I^{\prime}$. We define a partition $S_{1} \uplus \cdots \uplus S_{m}=\{1, \ldots, n\}$ for $I$ as follows. For each $k \in\{1, \ldots, m\}$, let

$S_{k}:=\left\{i \in\{1, \ldots, n\} \mid j_{i}^{1}\right.$ is scheduled on machine $\left.k\right\}$.

Since, for each $i \in\{1, \ldots, n\}$, the job $j_{i}^{1}$ is scheduled on exactly one machine, this is indeed a partition. We show that $\sum_{i \in S_{k}} a_{i} \leq V$ for each $k \in\{1, \ldots, m\}$. Assume, towards a contradiction, that there is a $k$ such that

$\sum_{i \in S_{k}} a_{i}>V$

By (3.1), for each $i \in\{1, \ldots, n\}$, the jobs in $J_{i}$ have the same release time, each has processing time at least $B$, and the length of the time window of each job is at most $B+A \leq$ $B+B / 2<2 B$. Thus, in any feasible schedule, the execution times of the $m$ jobs in $J_{i}$ mutually intersect. Hence, the jobs in $J_{i}$ are scheduled on $m$ mutually distinct machines. By the pigeonhole principle, for each $i \in\{1, \ldots, n\}$, exactly one job $j_{i}^{*} \in J_{i}$ is scheduled on machine $k$. We finish the proof by showing that,

$\forall i \in\{1, \ldots, n\}$, job $j_{i}^{*}$ is not finished before time $i B+\sum_{j \in S_{k}, j \leq i} a_{j}$.

This claim together with $\underline{(3.3)}$ then yields that job $j_{n}^{*}$ is not finished before

$n B+\sum_{j \in S_{k}, j \leq n} a_{j}=n B+\sum_{j \in S_{k}} a_{j}>n B+V$,

which contradicts the schedule being feasible, since jobs in $J_{n}$ have deadline $n B+V$ by (3.1). It remains to prove (3.4). We proceed by induction.

The earliest possible execution time of $j_{1}^{*}$ is, by (3.1), time 0 . The processing time of $j_{1}^{*}$ is $B$ if $j_{1}^{*} \neq j_{1}^{1}$, and $B \overline{+a_{1}}$ otherwise. By (3.2), $1 \in S_{k}$ if and only if $j_{1}^{1}$ is scheduled on machine $k$, that is, if and only if $j_{1}^{*}=j_{1}^{1}$. Thus, job $j_{1}^{*}$ is not finished before $B+\sum_{j \in S_{k}, j \leq i} a_{j}$ and (3.4) holds for $i=1$. Now, assume that (3.4) holds for $i-1$. We prove it for $i$. Since $j_{i-1}^{*}$ is not finished before $(i-1) B+\sum_{j \in S_{k}, j \leq i-1} a_{j}$, this is the earliest possible execution time of $j_{i}^{*}$. The processing time of $j_{i}^{*}$ is $B$ if $j_{i}^{*} \neq j_{i}^{1}$ and $B+a_{i}$ otherwise. By (3.2), $i \in S_{k}$ if and only if $j_{i}^{*}=j_{i}^{1}$. Thus, job $j_{i}^{*}$ is not finished before $i B+\sum_{j \in S_{k}, j \leq i} a_{j}$ and (3.4) holds.

We are now ready to finish the proof of Theorem $\underline{3.1}$.

Proof (of Theorem 3.1) By Lemmas 3.4 to 3.6, Construction 3.2 is a polynomial-time many-one reduction from BIN PACKING with $n \geq 2$ items and $m$ bins to $\lambda(m n)$-LOOSE INTERVAL-CONSTRAINEd SCHEDUling, where $\lambda: \mathbb{N} \rightarrow \mathbb{R}$ 
such that $\lambda(n) \geq 1+n^{-c}$ for some integer $c \geq 1$ and all $n \geq 2$. We now show the points (i) and (ii) of Theorem 3.1.

(i) follows since BIN PACKING is weakly NP-hard for $m=$ 2 (Jansen et al 2013) and since, by Lemma 3.4(i), Construction 3.2 outputs instances of $\lambda(m n)$-LOOSE INTERVAL-CONSTRAINED SCHEDULING with $m$ machines.

(ii) follows since BIN PACKING is W[1]-hard parameterized by $m$ even if the sizes of the $n$ items are bounded by a polynomial in $n$ (Jansen et al 2013). In this case, Construction 3.2 generates $\overline{\lambda(m n) \text {-LOOSE INTERVAL-CONSTRAINED }}$ SCHEDULING instances for which all numbers are bounded polynomially in the number of jobs by Lemma 3.5(ii). Moreover, Construction 3.2 maps the $m$ bins of the BIN PACKING instance to the $m$ machines of the output INTERVAL-CONSTRAINED SCHEDULING instance.

Concluding this section, it is interesting to note that Theorem 3.1 also shows W[1]-hardness of $\lambda$-LOOSE INTERVALCONSTRAINED SCHEDULING with respect to the height parameter considered by Cieliebak et al (2004):

Definition 3.7 (Height) For an INTERVAL-CONSTRAINED SCHEDULING instance and any time $t \in \mathbb{N}$, let

$S_{t}:=\left\{j \in J \mid t \in\left[t_{j}, d_{j}\right)\right\}$

denote the set of jobs whose time window contains time $t$. The height of an instance is

$h:=\max _{t \in \mathbb{N}}\left|S_{t}\right|$.

Proposition 3.8 Let $\lambda: \mathbb{N} \rightarrow \mathbb{R}$ be such that $\lambda(n) \geq 1+n^{-c}$ for some integer $c \geq 1$ and all $n \geq 2$.

Then $\lambda(n)$-LOOSE INTERVAL-CONSTRAINED SCHEDULING of $n$ jobs on m machines is W[1]-hard parameterized by the height $h$.

Proof Proposition 3.8 follows in the same way as Theorem 3.1; one additionally has to prove that Construction 3.2 outputs INTERVAL-CONSTRAINED SCHEDULING instances of height at most $2 m$. To this end, observe that, by (3.1), for each $i \in\{1, \ldots, n\}$, there are $m$ jobs released at time $(i-1) B$ whose deadline is no later than $i B+A<(i+1) B$ since $A \leq B / 2$. These are all jobs created by Construction 3.2. Thus, $S_{t}$ contains only the $m$ jobs released at time $\lfloor t / B\rfloor \cdot \bar{B}$ and the $m$ jobs released at time $\lfloor t / B-1\rfloor \cdot B$, which are $2 m$ jobs in total.

Remark 3.9 Proposition 3.8 complements findings of Cieliebak et al (2004), who provide a fixed-parameter tractability result for INTERVAL-CONSTRAINED SCHEDULING parameterized by $h+\sigma$ : our result shows that their algorithm presumably cannot be improved towards a fixed-parameter tractability result for INTERVAL-CONSTRAINED SCHEDULING parameterized by $h$ alone.

\section{An algorithm for bounded looseness}

In the previous section, we have seen that $\lambda$-LOOSE INTERVAL-CONSTRAINED SCHEDULING for any $\lambda>1$ is strongly W[1]-hard parameterized by $m$ and weakly NP-hard for $m=2$. We complement this result by the following theorem, which yields a pseudo-polynomial-time algorithm for each constant $m$ and $\lambda$.

Theorem 4.1 $\lambda$-Loose InTERVAL-CONSTRAINED SCHEDULING is solvable in $\ell^{O(\lambda m)} \cdot n+O(n \log n)$ time, where $\ell:=$ $\max _{j \in J}\left|d_{j}-t_{j}\right|$.

The crucial observation for the proof of Theorem 4.1 is the following lemma. It gives a logarithmic upper bound on the height $h$ of yes-instances (as defined in Definition 3.7). To prove Theorem 4.1, we will thereafter present an algorithm that has a running time that is single-exponential in $h$.

Lemma 4.2 Let I be a yes-instance of $\lambda$-LOOSE INTERVAL-CONSTRAINED SCHEDULING with $m$ machines and $\ell:=\max _{j \in J}\left|d_{j}-t_{j}\right|$. Then, I has height at most

$2 m \cdot\left(\frac{\log \ell}{\log \lambda-\log (\lambda-1)}+1\right)$.

Proof Recall from Definition 3.7 that the height of an INTERVAL-CONSTRAINED SCHEDULING instance is $\max _{t \in \mathbb{N}}\left|S_{t}\right|$.

We will show that, in any feasible schedule for $I$ and at any time $t$, there are at most $N$ jobs in $S_{t}$ that are active on the first machine at some time $t^{\prime} \geq t$, where

$N \leq \frac{\log \ell}{\log \lambda-\log (\lambda-1)}+1$.

By symmetry, there are at most $N$ jobs in $S_{t}$ that are active on the first machine at some time $t^{\prime} \leq t$. Since there are $m$ machines, the total number of jobs in $S_{t}$ at any time $t$, and therefore the height, is at most $2 m N$.

It remains to show (4.1). To this end, fix an arbitrary time $t$ and an arbitrary feasible schedule for $I$. Then, for any $d \geq 0$, let $J(t+d) \subseteq S_{t}$ be the set of jobs that are active on the first machine at some time $t^{\prime} \geq t$ but finished by time $t+d$. We show by induction on $d$ that

$|J(t+d)| \leq \begin{cases}0 & \text { if } d=0 \\ -\frac{\log d}{\log (1-1 / \lambda)}+1 & \text { if } d \geq 1 .\end{cases}$

If $d=0$, then $|J(t+0)|=0$ and (4.2) holds. Now, consider

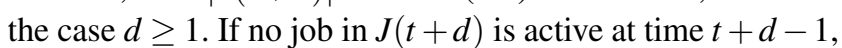
then $J(t+d)=J(t+d-1)$ and (4.2) holds by the induction hypothesis. Now, assume that there is a job $j \in J(t+d)$ that is active at time $t+d-1$. Then, $d_{j} \geq t+d$ and, since $j \in S_{t}$, $t_{j} \leq t$. Hence,

$p_{j} \geq \frac{\left|d_{j}-t_{j}\right|}{\lambda} \geq \frac{|t+d-t|}{\lambda}=\frac{d}{\lambda}$. 
It follows that

$|J(t+d)| \leq 1+|J(t+d-\lceil d / \lambda\rceil)|$.

Thus, if $d-\lceil d / \lambda\rceil=0$, then $|J(t+d)| \leq 1+|J(t)| \leq 1$ and (4.2) holds. If $d-\lceil d / \lambda\rceil>0$, then, by the induction hypothesis, the right-hand side of $\underline{(4.3)}$ is

$$
\begin{aligned}
& \leq 1-\frac{\log (d-\lceil d / \lambda\rceil)}{\log (1-1 / \lambda)}+1 \\
& \leq 1-\frac{\log (d(1-1 / \lambda))}{\log (1-1 / \lambda)}+1 \\
& =1-\frac{\log d+\log (1-1 / \lambda)}{\log (1-1 / \lambda)}+1 \\
& =-\frac{\log d}{\log (1-1 / \lambda)}+1
\end{aligned}
$$

and (4.2) holds. Finally, since $\ell=\max _{1 \leq j \leq n}\left|d_{j}-t_{j}\right|$, no job in $S_{t}$ is active at time $t+\ell$. Hence, we can now prove (4.1) using (4.2) by means of

$$
\begin{aligned}
N \leq|J(t+\ell)| & \leq-\frac{\log \ell}{\log (1-1 / \lambda)}+1 \\
& =-\frac{\log \ell}{\log \left(\frac{\lambda-1}{\lambda}\right)}+1 \\
& =-\frac{\log \ell}{\log (\lambda-1)-\log \lambda}+1 \\
& =\frac{\log \ell}{\log \lambda-\log (\lambda-1)}+1 .
\end{aligned}
$$

The following proposition gives some intuition on how the bound behaves for various $\lambda$.

Proposition 4.3 For any $\lambda \geq 1$ and any $b \in(1, e]$, it holds that

$$
\frac{1}{\log _{b} \lambda-\log _{b}(\lambda-1)} \leq \lambda
$$

Proof It is well-known that $(1-1 / \lambda)^{\lambda}<1 / e$ for any $\lambda \geq 1$. Hence, $\lambda \log _{b}(1-1 / \lambda)=\log _{b}(1-1 / \lambda)^{\lambda}<\log _{b} 1 / e \leq-1$, that is, $-\lambda \log _{b}(1-1 / \lambda) \geq 1$. Thus,

$\frac{1}{-\lambda \log _{b}(1-1 / \lambda)} \leq 1$ and $\frac{1}{-\log _{b}(1-1 / \lambda)} \leq \lambda$.

Finally,

$$
\begin{aligned}
\frac{1}{-\log _{b}(1-1 / \lambda)} & =\frac{1}{-\log _{b}\left(\frac{\lambda-1}{\lambda}\right)} \\
& =\frac{1}{-\log _{b}(\lambda-1)+\log _{b} \lambda} .
\end{aligned}
$$

Towards our proof of Theorem 4.1 , Lemma 4.2 provides a logarithmic upper bound on the height $h$ of yes-instances of INTERVAL-CONSTRAINED SCHEDULING. Our second step towards the proof of Theorem 4.1 is the following algorithm, which runs in time that is single-exponential in $h$. We first present the algorithm and, thereafter, prove its correctness and running time.
Algorithm 4.4 We solve InTERVAL-CONSTRAINED SCHEDULING using dynamic programming. First, for an INTERVALCONSTRAINED SCHEDULING instance, let $\ell:=\max _{j \in J} \mid d_{j}-$ $t_{j} \mid$, let $S_{t}$ be as defined in Definition 3.7, and let $S_{t}^{<} \subseteq J$ be the set of jobs $j$ with $d_{j} \leq t$, that is, that have to be finished by time $t$.

We compute a table $T$ that we will show to have the following semantics. For a time $t \in \mathbb{N}$, a subset $S \subseteq S_{t}$ of jobs and a vector $\mathbf{b}=\left(b_{1}, \ldots, b_{m}\right) \in\{-\ell, \ldots, \ell\}^{m}$,

$T[t, S, \mathbf{b}]= \begin{cases}1 \quad & \text { if all jobs in } S \cup S_{t}^{<} \text {can be scheduled so } \\ & \text { that machine } i \text { is idle from time } t+b_{i} \\ & \text { for each } i \in\{1, \ldots, m\} \\ 0 \quad & \text { otherwise. }\end{cases}$

To compute $T$, first, set $T[0, \emptyset, \mathbf{b}]:=1$ for every vector $\mathbf{b} \in$ $\{-\ell, \ldots, \ell\}^{m}$. Now we compute the other entries of $T$ by increasing $t$, for each $t$ by increasing $\mathbf{b}$, and for each $\mathbf{b}$ by $S$ with increasing cardinality. Herein, we distinguish two cases.

(a) If $t \geq 1$ and $S \subseteq S_{t-1}$, then set $T[t, S, \mathbf{b}]:=T\left[t-1, S^{\prime}, \mathbf{b}^{\prime}\right]$, where

$$
\begin{aligned}
& S^{\prime}:=S \cup\left(S_{t-1} \cap S_{t}^{<}\right) \text {and } \mathbf{b}^{\prime}:=\left(b_{1}^{\prime}, \ldots, b_{m}^{\prime}\right) \text { with } \\
& b_{i}^{\prime}:=\min \left\{b_{i}+1, \ell\right\} \text { for each } i \in\{1, \ldots, m\} .
\end{aligned}
$$

(b) Otherwise, set $T[t, S, \mathbf{b}]:=1$ if and only if at least one of the following two cases applies:

i) there is a machine $i \in\{1, \ldots, m\}$ such that $b_{i}>-\ell$ and $T\left[t, S, \mathbf{b}^{\prime}\right]=1$, where $\mathbf{b}^{\prime}:=\left(b_{1}^{\prime}, \ldots, b_{m}^{\prime}\right)$ with

$$
b_{i^{\prime}}^{\prime}:= \begin{cases}b_{i}-1 & \text { if } i^{\prime}=i \\ b_{i^{\prime}} & \text { if } i^{\prime} \neq i\end{cases}
$$

or

ii) there is a job $j \in S$ and a machine $i \in\{1, \ldots, m\}$ such that $b_{i}>0, t+b_{i} \leq d_{j}, t+b_{i}-p_{j} \geq t_{j}$, and $T\left[t, S \backslash\{j\}, \mathbf{b}^{\prime}\right]=1$, where $\mathbf{b}^{\prime}:=\left(b_{1}^{\prime}, \ldots, b_{m}^{\prime}\right)$ with

$b_{i^{\prime}}^{\prime}:= \begin{cases}b_{i}-p_{j} & \text { if } i^{\prime}=i, \\ b_{i^{\prime}} & \text { if } i^{\prime} \neq i .\end{cases}$

Note that, since $j \in S_{t}$, one has $t_{j} \geq t-\ell$ by definition of $\ell$. Hence, $b_{i}^{\prime} \geq-\ell$ is within the allowed range $\{-\ell, \ldots, \ell\}$.

Finally, we answer yes if and only if $T\left[t_{\max }, S_{t_{\max }}, \mathbf{1}^{m} \cdot \ell\right]=1$, where $t_{\max }:=\max _{j \in J} t_{j}$.

Lemma 4.5 Algorithm 4.4 correctly decides INTERVAL-CONSTRAINED SCHEDULING.

Proof We prove the following two claims: For any time $0 \leq$ $t \leq t_{\text {max }}$, any set $S \subseteq S_{t}$, and any vector $\mathbf{b}=\left(b_{1}, \ldots, b_{m}\right) \in$ $\{-\ell, \ldots, \ell\}^{m}$

if $T[t, S, \mathbf{b}]=1$, then all jobs in $S \cup S_{t}^{<}$can be scheduled so that machine $i$ is idle from time $t+b_{i}$ for each $i \in\{1, \ldots, m\}$, 
and

if all jobs in $S \cup S_{t}^{<}$can be scheduled so that machine $i$ is idle from time $t+b_{i}$ for each $i \in$ $\{1, \ldots, m\}$, then $T[t, S, \mathbf{b}]=1$.

From (4.4) and (4.5), the correctness of the algorithm easily follows: observe that, in any feasible schedule, all machines are idle from time $t_{\max }+\ell$ and all jobs $J \subseteq S_{t_{\max }} \cup S_{t_{\max }}^{<}$are scheduled. Hence, there is a feasible schedule if and only if $T\left[t_{\max }, S_{t_{\max }}, \mathbf{1}^{m} \cdot \ell\right]=1$. It remains to prove (4.4) and (4.5) .

First, we prove (4.4) by induction. For $T[0, \emptyset, \mathbf{b}]=1$, (4.4) holds since there are no jobs to schedule. We now prove (4.4) for $T[t, S, \mathbf{b}]$ under the assumption that it is true for all $\left.\overline{T\left[t^{\prime}\right.}, S^{\prime}, \mathbf{b}^{\prime}\right]$ with $t^{\prime}<t$ or $t^{\prime}=t$ and $\mathbf{b}^{\prime} \supsetneqq \mathbf{b}$.

If $T[t, S, \mathbf{b}]$ is set to 1 in Algorithm 4.4(a), then, for $S^{\prime}$ and $\mathbf{b}^{\prime}$ as defined in Algorithm 4.4(a), $\left.\overline{T[t}-1, S^{\prime}, \mathbf{b}^{\prime}\right]=1$. By the induction hypothesis, all jobs in $S^{\prime} \cup S_{t-1}^{<}$can be scheduled so that machine $i$ is idle from time $t-1+b_{i}^{\prime} \leq$ $t+b_{i}$. Moreover, $S \cup S_{t}^{<}=S^{\prime} \cup S_{t-1}^{<}$since $S^{\prime}=S \cup\left(S_{t-1} \cap S_{t}^{<}\right)$. Hence, (4.4) follows.

If $T[t, S, \mathbf{b}]$ is set to 1 in Algorithm 4.4(bi), then one has $T\left[t, S, \mathbf{b}^{\prime}\right]=1$ for $\mathbf{b}^{\prime}$ as defined in Algorithm 4.4(bi). By the induction hypothesis, all jobs in $S \cup S_{t}^{<}$can be scheduled so that machine $i^{\prime}$ is idle from time $t+b_{i^{\prime}}^{\prime} \leq t+b_{i^{\prime}}$, and (4.4) follows.

If $T[t, S, \mathbf{b}]$ is set to 1 in Algorithm 4.4(bii), then $T[t, S \backslash$ $\left.\{j\}, \mathbf{b}^{\prime}\right]=1$ for $j$ and $\mathbf{b}^{\prime}$ as defined in Algorithm 4.4(bii). By the induction hypothesis, all jobs in $(S \backslash\{j\}) \cup \overline{S_{t}^{<}} \overline{\text { can }}$ be scheduled so that machine $i^{\prime}$ is idle from time $t+b_{i^{\prime}}^{\prime}$. It remains to schedule job $j$ on machine $i$ in the interval $[t+$ $\left.b_{i}^{\prime}, t+b_{i}\right)$, which is of length exactly $p_{j}$ by the definition of $\mathbf{b}^{\prime}$. Then, machine $i$ is idle from time $t+b_{i}$ and any machine $i^{\prime} \neq i$ is idle from time $t+b_{i^{\prime}}^{\prime}=t+b_{i^{\prime}}$, and (4.4) follows.

It remains to prove (4.5). We use induction. Claim (4.5) clearly holds for $t=0, \overline{S=\emptyset}$, and any $\mathbf{b} \in\{-\ell, \ldots, \ell\}^{\bar{m} \text { by }}$ the way Algorithm 4.4 initializes $T$. We now show (4.5) provided that it is true for $t^{\prime}<t$ or $t^{\prime}=t$ and $\mathbf{b}^{\prime} \supsetneqq \mathbf{b}$.

If $S \subseteq S_{t-1}$, then $S \cup S_{t}^{<}=S^{\prime} \cup S_{t-1}^{<}$for $S^{\prime}$ as defined in Algorithm 4.4(a). Moreover, since no job in $S^{\prime} \cup S_{t-1}^{<}$can be active from time $t-1+\ell$ by definition of $\ell$, each machine $i$ is idle from time $t-1+\min \left\{b_{i}+1, \ell\right\}=t-1+b_{i}^{\prime}$, for $\mathbf{b}^{\prime}=$ $\left(b_{1}^{\prime}, \ldots, b_{m}^{\prime}\right)$ as defined in Algorithm 4.4(a). Hence, $T[t-$ $\left.1, S^{\prime}, \mathbf{b}^{\prime}\right]=1$ by the induction hypothesis, Algorithm 4.4(a) applies, sets $T[t, S, \mathbf{b}]:=T\left[t-1, S^{\prime}, \mathbf{b}^{\prime}\right]=1$, and (4.5) holds.

If some machine $i$ is idle from time $t+b_{i}-\overline{1}$, then, by the induction hypothesis, $T\left[t, S, \mathbf{b}^{\prime}\right]=1$ in Algorithm 4.4(bi), the algorithm sets $T[t, S, \mathbf{b}]:=1$, and (4.5) holds.

In the remaining case, every machine $i$ is busy at time $t+$ $b_{i}-1$ and $K:=S \backslash S_{t-1} \neq \emptyset$. Thus, there is a machine $i$ executing a job from $K$. For each job $j^{\prime} \in K$, we have $t_{j^{\prime}} \geq t$. Since machine $i$ is idle from time $t+b_{i}$ and executes $j^{\prime}$, one has $b_{i}>0$. Let $j$ be the last job scheduled on machine $i$. Then, since machine $i$ is busy at time $t+b_{i}-1$, we have $d_{j} \geq t+$ $b_{i}>t$ and $j \notin S_{t}^{<}$. Hence, $j \in S_{t}$. Since machine $i$ is idle from time $t+b_{i}$, we also have $t+b_{i}-p_{j} \geq t_{j}$. Now, if we remove $j$ from the schedule, then machine $i$ is idle from time $t+b_{i}-p_{j}$ and each machine $i^{\prime} \neq i$ is idle from time $t+b_{i^{\prime}}^{\prime}=t+b_{i^{\prime}}$. Thus, by the induction hypothesis, $T\left[t, S \backslash\{j\}, \mathbf{b}^{\prime}\right]=1$ in Algorithm 4.4(bii), the algorithm sets $T[t, S, \mathbf{b}]:=1$, and (4.5) holds.

Lemma 4.6 Algorithm 4.4 can be implemented to run in $O\left(2^{h} \cdot(2 \ell+1)^{m} \cdot\left(h^{2} m+\overline{h m}^{2}\right) \cdot n \ell+n \log n\right)$ time, where $\ell:=$ $\max _{j \in J}\left|d_{j}-t_{j}\right|$ and $h$ is the height of the input instance.

Proof Concerning the running time of Algorithm 4.4, we first bound $t_{\max }$. If $t_{\max }>n \ell$, then there is a time $t \in\left\{\overline{\left.0, \ldots, t_{\max }\right\}}\right.$ such that $S_{t}=\emptyset$ (cf. Definition 3.7). Then, we can split the instance into one instance with the jobs $S_{t}^{<}$and into one instance with the jobs $J \backslash S_{t}^{<}$. We answer "yes" if and only if both of them are yes-instances. Henceforth, we assume that $t_{\max } \leq n \ell$.

In a preprocessing step, we compute the sets $S_{t}$ and $S_{t-1} \cap$ $S_{t}^{<}$, which can be done in $O\left(n \log n+h n+t_{\max }\right)$ time by sorting the input jobs by deadlines and scanning over the input time windows once: if no time window starts or ends at time $t$, then $S_{t}$ is simply stored as a pointer to the $S_{t^{\prime}}$ for the last time $t^{\prime}$ where a time window starts or ends.

Now, the table $T$ of Algorithm 4.4 has at most $\left(t_{\max }+\right.$ $1) \cdot 2^{h} \cdot(2 \ell+1)^{m} \leq(n \ell+1) \cdot 2^{h} \cdot(2 \overline{\ell+1})^{m}$ entries. A table entry $T[t, S, \mathbf{b}]$ can be accessed in $O(m+h)$ time using a carefully initialized trie data structure (van Bevern 2014) since $|S| \leq h$ and since $\mathbf{b}$ is a vector of length $m$.

To compute an entry $T[t, S, \mathbf{b}]$, we first check, for each job $j \in S$, whether $j \in S_{t-1}$. If this is the case for each $j$, then Algorithm 4.4(a) applies. We can prepare $\mathbf{b}^{\prime}$ in $O(m)$ time and $S^{\prime}$ in $\overline{O(h)}$ time using the set $S_{t-1} \cap S_{t}^{<}$computed in the preprocessing step. Then, we access the entry $T\left[t-1, S^{\prime}, \mathbf{b}^{\prime}\right]$ in $O(h+m)$ time. Hence, (a) takes $O(h+m)$ time.

If Algorithm 4.4(a) does not apply, then we check whether Algorithm 4.4(bi) applies. To this end, for each $i \in\{1, \ldots, m\}$, we prepare $\overline{\mathbf{b}^{\prime}}$ in $O(m)$ time and access $T\left[t, S, \mathbf{b}^{\prime}\right]$ in $O(h+m)$ time. Hence, it takes $O\left(m^{2}+h m\right)$ time to check (bi).

To check whether Algorithm 4.4(bii) applies, we try each $j \in S$ and each $i \in\{1, \ldots, m\}$ and, for each, prepare $\mathbf{b}^{\prime}$ in $O(m)$ time and check $T\left[t, S \backslash\{j\}, \mathbf{b}^{\prime}\right]$ in $O(h+m)$ time. Thus (bii) can be checked in $O\left(h^{2} m+h m^{2}\right)$ time.

With the logarithmic upper bound on the height $h$ of yesinstances of INTERVAL-CONSTRAINED SCHEDULING given by Lemma 4.2 and using Algorithm 4.4, which, by Lemma 4.6, runs in time that is single-exponential in $h$ for a fixed number $m$ of machines, we can now prove Theorem 4.1 .

Proof (of Theorem 4.1) We use the following algorithm. Let $h:=2 m \cdot\left(\frac{\log \ell}{\log \lambda-\log (\lambda-1)}+1\right)$. 
If, for any time $t \in \mathbb{N}$, we have $\left|S_{t}\right|>h$, then we are facing a no-instance by Lemma 4.2 and immediately answer "no". This can be checked in $O \overline{(n \log n)}$ time: one uses the interval graph coloring problem to check whether we can schedule the time windows of all jobs (as intervals) onto $h$ machines.

Otherwise, we conclude that our input instance has height at most $h$. We now apply Algorithm 4.4, which, by Lemma 4.6, runs in $O\left(2^{h} \cdot(2 \ell+1)^{m} \cdot\left(h^{2} m+\overline{h m^{2}}\right) \cdot n \ell+n \log n\right)$ time. Since, by Proposition 4.3, $h \in O(\lambda m \log \ell)$, this running time is $\ell^{O(\lambda m)} h \cdot n+O(n \log n)$.

A natural question is whether Theorem 4.1 can be generalized to $\lambda=\infty$, that is, to INTERVAL-CONSTRAINED SCHEDULING without looseness constraint. This question can be easily answered negatively using a known reduction from 3-PARTITION to INTERVAL-CONSTRAINED SCHEDULING given by Garey and Johnson (1979):

Proposition 4.7 If there is an $\ell^{O(m)} \cdot \operatorname{poly}(n)$-time algorithm for INTERVAL-CONSTRAINED SCHEDULING, where $\ell:=$ $\max _{j \in J}\left|d_{j}-t_{j}\right|$, then $P=N P$.

Proof Garey and Johnson (1979, Theorem 4.5) showed that INTER VAL-CONSTRAINED SCHEDULING is NP-hard even on $m=1$ machine. In their reduction, $\ell \in \operatorname{poly}(n)$. A supposed $\ell^{O(m)} \cdot \operatorname{poly}(n)$-time algorithm would solve such instances in polynomial time.

\section{An algorithm for bounded slack}

So far, we considered INTERVAL-CONSTRAINED SCHEDULING with bounded looseness $\lambda$. Cieliebak et al (2004) additionally considered INTER VAL-CONSTRAINED SCHEDULING for any constant slack $\sigma$.

Recall that Cieliebak et al (2004) showed that $\lambda$-LoOSE INTERVAL-CONSTRAINED SCHEDULING is NP-hard for any constant $\lambda>1$ and that Theorem $\underline{3.1}$ shows that having a small number $m$ of machines does make the problem significantly easier.

Similarly, Cieliebak et al (2004) showed that $\sigma$-SLACK INTERVAL-CONSTRAINED SCHEDULING is NP-hard already for $\sigma=2$. Now we contrast this result by showing that $\sigma$-SLACK INTERVAL-CONSTRAINED SCHEDULING is fixed-parameter tractable for parameter $m+\sigma$. More specifically, we show the following:

Theorem 5.1 $\sigma$-SLACK INTERVAL-CONSTRAINED SCHEDULING is solvable in time

$O\left((\sigma+1)^{(2 \sigma+1) m} \cdot n \cdot \sigma m \cdot \log \sigma m+n \log n\right)$.

Similarly as in the proof of Theorem $\underline{4.1}$, we first give an upper bound on the height of yes-instances of INTERVALConstrained Scheduling as defined in Definition $\underline{3.7}$. To this end, we first show that each job $j \in S_{t}$ has to occupy some of the (bounded) machine resources around time $t$.
Lemma 5.2 At any time $t$ in any feasible schedule for $\sigma$ SLACK INTERVAL-CONSTRAINED SCHEDULING, each job $j \in S_{t}$ is active at some time in the interval $[t-\sigma, t+\sigma]$.

Proof If the time window of $j$ is entirely contained in $[t-$ $\sigma, t+\sigma]$, then, obviously, $j$ is active at some time during the interval $[t-\sigma, t+\sigma]$.

Now, assume that the time window of $j$ is not contained in $[t-\sigma, t+\sigma]$. Then, since $j \in S_{t}$, its time window contains $t$ by Definition 3.7 and, therefore, one of $t-\sigma$ or $t+\sigma$. Assume, for the sake of contradiction, that there is a schedule such that $j$ is not active during $[t-\sigma, t+\sigma]$. Then $j$ is inactive for at least $\sigma+1$ time units in its time windowa contradiction.

Now that we know that each job in $S_{t}$ has to occupy machine resources around time $t$, we can bound the size of $S_{t}$ in the amount of resources available around that time.

Lemma 5.3 Any yes-instance of $\sigma$-SLACK INTERVAL-CONSTRAINED SCHEDULING has height at most $(2 \sigma+1) m$.

Proof Fix any feasible schedule for an arbitrary yes-instance of $\sigma$-SLACK INTERVAL-CONSTRAINED SCHEDULING and any time $t$. By Lemma 5.2, each job in $S_{t}$ is active at some time in the interval $[t-\overline{\sigma, t}+\sigma]$. This interval has length $2 \sigma+$ 1 . Thus, on $m$ machines, there is at most $(2 \sigma+1) m$ available processing time in this time interval. Consequently, there can be at most $(2 \sigma+1) m$ jobs with time intervals in $S_{t}$.

We finally arrive at the algorithm to prove Theorem 5.1.

Proof (of Theorem 5.1) Let $h:=(2 \sigma+1) m$. In the same way as for Theorem $\overline{4.1}$, in $O(n \log n)$ time we discover that we face a no-instance due to Lemma $\underline{5.3}$ or, otherwise, that our input instance has height at most $h$. In the latter case, we apply the $O\left(n \cdot(\sigma+1)^{h} \cdot h \log h\right)$-time algorithm due to Cieliebak et al (2004).

\section{Conclusion}

Despite the fact that there are comparatively few studies on the parameterized complexity of scheduling problems, the field of scheduling indeed offers many natural parameterizations and fruitful challenges for future research. Notably, Marx (2011) saw one reason for the lack of results on "parameterized scheduling" in the fact that most scheduling problems remain NP-hard even for a constant number of machines (a very obvious and natural parameter indeed), hence destroying hope for fixed-parameter tractability results with respect to this parameter. In scheduling interval-constrained jobs with small looseness and small slack, we also have been confronted with this fact, facing (weak) NP-hardness even for two machines. 
The natural way out of this misery, however, is to consider parameter combinations, for instance combining the parameter number of machines with a second one. In our study, these were combinations with looseness and with slack (see also Table 1.1). In a more general perspective, this consideration makes scheduling problems a prime candidate for offering a rich set of research challenges in terms of a multivariate complexity analysis (Fellows et al 2013; Niedermeier 2010). Herein, for obtaining positive algorithmic results, research has to go beyond canonical problem parameters, since basic scheduling problems remain NP-hard even if canonical parameters are simultaneously bounded by small constants, as demonstrated by Kononov et al (2012). ${ }^{1}$

Natural parameters to be studied in future research on INTERVAL-CONSTRAINED SCHEDULING are the combination of slack and looseness - the open field in our Table 1.1—and the maximum and minimum processing times, which were found to play an important role in the online version of the problem (Saha 2013).

Finally, we point out that our fixed-parameter algorithms for INTERVAL-CONSTRAINED SCHEDULING are easy to implement and may be practically applicable if the looseness, slack, and number of machines is small (about three or four each). Moreover, our algorithms are based on upper bounds on the height of an instance in terms of its number of machines, its looseness, and slack. Obviously, this can also be exploited to give lower bounds on the number of required machines based on the structure of the input instance, namely, on its height, looseness, and slack. These lower bounds may be of independent interest in exact branch and bound or approximation algorithms for the machine minimization problem.

\section{References}

van Bevern R (2014) Towards optimal and expressive kernelization for $d$-Hitting Set. Algorithmica 70(1):129-147

van Bevern R, Chen J, Hüffner F, Kratsch S, Talmon N, Woeginger GJ (2015a) Approximability and parameterized complexity of multicover by $c$-intervals. Information Processing Letters 115(10):744749

van Bevern R, Mnich M, Niedermeier R, Weller M (2015b) Interval scheduling and colorful independent sets. Journal of Scheduling 18(5):449-469

Bodlaender HL, Fellows MR (1995) W[2]-hardness of precedence constrained $k$-processor scheduling. Operations Research Letters 18(2):93-97

Chen L, Megow N, Schewior K (2016) An $O(\log m)$-competitive algorithm for online machine minimization. In: Proceedings of the 27th Annual ACM-SIAM Symposium on Discrete Algorithms (SODA'16), SIAM, pp 155-163

\footnotetext{
1 The results of Kononov et al (2012) were obtained in context of a multivariate complexity analysis framework described by Sevastianov (2005), which is independent of the framework of parameterized complexity theory considered in our work: it allows for systematic classification of problems as polynomial-time solvable or NP-hard given concrete constraints on a set of instance parameters. It is plausible that this framework is applicable to classify problems as FPT or W[1]-hard as well.
}

Chuzhoy J, Guha S, Khanna S, Naor J (2004) Machine minimization for scheduling jobs with interval constraints. In: Proceedings of the 45th Annual Symposium on Foundations of Computer Science (FOCS'04), pp 81-90

Cieliebak M, Erlebach T, Hennecke F, Weber B, Widmayer P (2004) Scheduling with release times and deadlines on a minimum number of machines. In: Exploring New Frontiers of Theoretical Informatics, IFIP International Federation for Information Processing, vol 155 , Springer, pp 209-222

Cygan M, Fomin FV, Kowalik L, Lokshtanov D, Marx D, Pilipczuk M, Pilipczuk M, Saurabh S (2015) Parameterized Algorithms. Springer

Downey RG, Fellows MR (2013) Fundamentals of Parameterized Complexity. Springer

Fellows MR, McCartin C (2003) On the parametric complexity of schedules to minimize tardy tasks. Theoretical Computer Science 298(2):317-324

Fellows MR, Jansen BMP, Rosamond FA (2013) Towards fully multivariate algorithmics: Parameter ecology and the deconstruction of computational complexity. European Journal of Combinatorics 34(3):541-566

Flum J, Grohe M (2006) Parameterized Complexity Theory. Springer

Garey MR, Johnson DS (1979) Computers and Intractability: A Guide to the Theory of NP-Completeness. Freeman

Halldórsson MM, Karlsson RK (2006) Strip graphs: Recognition and scheduling. In: Proceedings of the 32nd International Workshop on Graph-Theoretic Concepts in Computer Science (WG'06), Springer, LNCS, vol 4271, pp 137-146

Hermelin D, Kubitza JM, Shabtay D, Talmon N, Woeginger G (2015) Scheduling two competing agents when one agent has significantly fewer jobs. In: Proceedings of the 10th International Symposium on Parameterized and Exact Computation (IPEC'15), Leibniz International Proceedings in Informatics (LIPIcs), vol 43, Schloss Dagstuhl-Leibniz-Zentrum für Informatik, pp 55-65

Jansen K, Kratsch S, Marx D, Schlotter I (2013) Bin packing with fixed number of bins revisited. Journal of Computer and System Sciences 79(1):39-49

Kolen AWJ, Lenstra JK, Papadimitriou CH, Spieksma FCR (2007) Interval scheduling: A survey. Naval Research Logistics 54(5):530543

Kononov A, Sevastyanov S, Sviridenko M (2012) A complete 4parametric complexity classification of short shop scheduling problems. Journal of Scheduling 15(4):427-446

Malucelli F, Nicoloso S (2007) Shiftable intervals. Annals of Operations Research 150(1):137-157

Marx D (2011) Fixed-parameter tractable scheduling problems. In: Packing and Scheduling Algorithms for Information and Communication Services (Dagstuhl Seminar 11091)

Mnich M, Wiese A (2015) Scheduling and fixed-parameter tractability. Mathematical Programming 154(1-2):533-562

Niedermeier R (2006) Invitation to Fixed-Parameter Algorithms. Oxford University Press

Niedermeier R (2010) Reflections on multivariate algorithmics and problem parameterization. In: Proceedings of the 27th International Symposium on Theoretical Aspects of Computer Science (STACS'10), Schloss Dagstuhl-Leibniz-Zentrum für Informatik, Leibniz International Proceedings in Informatics (LIPIcs), vol 5, pp 17-32

Saha B (2013) Renting a cloud. In: Annual Conference on Foundations of Software Technology and Theoretical Computer Science (FSTTCS) 2013, Schloss Dagstuhl-Leibniz-Zentrum für Informatik, Leibniz International Proceedings in Informatics (LIPIcs), vol 24, pp 437-448

Sevastianov SV (2005) An introduction to multi-parameter complexity analysis of discrete problems. European Journal of Operational Research 165(2):387-397 